\title{
Las TIC como mediadoras en el proceso enseñanza - aprendizaje durante la pandemia del COVID-19
}

\section{ICT as mediators in the teaching-learning process during the COVID-19 pandemic}

\author{
Paola Cecilia Pinos-Coronel \\ paola.pinos@psg.ucacue.edu.ec \\ Universidad Católica de Cuenca, Azogues \\ Ecuador \\ https://orcid.org/0000-0003-4770-9116 \\ Darwin Gabriel García-Herrera \\ dggarciah@ucacue.edu.ec \\ Universidad Católica de Cuenca, Azogues \\ Ecuador \\ https://orcid.org/0000-0001-6813-8100 \\ Juan Carlos Erazo-Álvarez \\ jcerazo@ucacue.edu.ec \\ Universidad Católica de Cuenca, Cuenca \\ Ecuador \\ https://orcid.org/0000-0001-6480-2270 \\ Cecilia Ivonne Narváez-Zurita \\ inarvaez@ucacue.edu.ec \\ Universidad Católica de Cuenca, Cuenca \\ Ecuador \\ https://orcid.org/0000-0002-7437-9880
}

Recibido: 21 de abril de 2020

Revisado: 04 de mayo de 2020

Aprobado: 25 de mayo de 2020

Publicado: 22 de junio de 2020 


\title{
RESUMEN
}

El objetivo de la investigación fue identificar el uso y manejo de herramientas tecnológicas en el proceso enseñanza - aprendizaje durante la pandemia de Coronavirus COVID-19 en Ecuador, mediante el análisis de la utilización de recursos TIC. Para ello se realizó la investigación descriptiva de cohorte trasversal. De este modo se observó que las TIC son el medio fundamental para la enseñanza, sobre todo para llevar a cabo la teleeducación durante la pandemia Covid-19, pese a que educadores y educandos no estuvieron preparados para este cambio radical de abandonar las clases presenciales y pasar a modo virtual, se ha hecho lo posible por alcanzar una conexión profesor - estudiante, buscando las herramientas tecnológicas que permitan y faciliten brindar una buena educación con el único fin de interactuar y trasmitir conocimientos para alcanzar una educación significativa.

Descriptores: Tecnología de la información; tecnología educacional; aprendizaje activo; epidemia. (Palabras del Tesauro UNESCO).

\begin{abstract}
The objective of the research was to identify the use and management of technological tools in the teaching-learning process during the Coronavirus COVID-19 pandemic in Ecuador, by analyzing the use of ICT resources. For this, the descriptive research of the transversal cohort was carried out. In this way, it was observed that ICTs are the fundamental means for teaching, especially to carry out teleeducation during the Covid-19 pandemic, despite the fact that educators were not prepared for this radical change of leaving face-to-face classes and Going into virtual mode, every effort has been made to achieve a teacher-student connection, seeking the technological tools that allow and facilitate providing a good education for the sole purpose of interacting and transmitting knowledge to achieve a meaningful education.
\end{abstract}

Descriptors: Information technology; educational technology; activity learning; epidemics. (Words from the UNESCO Thesaurus).

\section{INTRODUCCIÓN}

Las Tecnologías de la Información y Comunicación (TIC), alcanzaron gran desarrollo a finales del siglo XX y al comienzo del siglo XXI, propiciando la llamada "Sociedad del Conocimiento" o "de la Información". Este desarrollo sin duda está presente en todos los ámbitos del ser humano ya sea en el gobierno, la educación, la salud, los negocios, las finanzas, las comunicaciones, la industria, es decir en el 
desenvolvimiento común y diario de toda persona (Organización de las Naciones Unidas para la Educación, la Ciencia y la Cultura, 2013).

Tradicionalmente, el acceso a la información era una de las mayores dificultades en la educación, debido a que se debía asistir a una biblioteca cercana o buscar información en libros y revistas antiguas. Con el surgimiento de las TIC, la disponibilidad de la información es abundante y el reto principal está en la selección, integración curricular y adquisición de competencias, para la utilización y manejo de la información. Pero lo que quizás sea valioso para la enseñanza de las ciencias, es que los ordenadores, tablets, smartphone, proyectores y otros dispositivos, se transformen en poderosas herramientas que facilitan explorar acontecimientos naturales y culturales, para utilizarlos en las aulas de clase y laboratorios con el fin de realizar interesantes experimentos con distintos grados de dificultad y desafíos (Quevedo-Álava, et al., 2020).

En la actualidad la sociedad se encuentra inmersa en avances científicos y tecnológicos, en el ámbito educativo se ha generalizado la utilización de las TIC, lo cual ha permitido optimizar el proceso enseñanza - aprendizaje, según (Morffe, 2010) la aplicación de nuevas metodologías y estrategias como el constructivismo y el colaboracionismo, que consideran como herramienta mediadora el uso de las TIC; específicamente en la búsqueda y selección de información, el análisis crítico y la resolución de problemas.

La aparición de las redes de telecomunicaciones y el internet han dado un vuelco radical al proceso de enseñanza y por ende al aprendizaje de las personas, debido a la gran cantidad de las fuentes de información existentes, permitiendo que los usuarios intercambien datos de todo tipo (Saltos-Cedeño, Vallejo-Valdivieso \& Moya-Martínez, 2020). Algunos de los avances tecnológicos como: las redes sociales, internet móvil, el cloud y cloud computing, han transformando las formas de comunicación, aprendizaje y la interacción entre usuarios (Orduz, 2012). Algunas herramientas como son los blogs, las redes sociales, los wikis, han generado nuevos espacios virtuales de aprendizaje forma y no formal.

Según (García-Martín \& Cantón-Mayo, 2019), el uso del ordenador contribuye significativamente en la formación del estudiante, en vista a los resultados obtenidos 
en su investigación mostraron que los adolescentes utilizaban herramientas tecnológicas, puesto que nueve de cada diez estudiantes realizaban búsquedas en Internet, interactúan y comparten audios y videos, consultaban información en wikis, tutoriales y YouTube pero sobre todo el uso de redes sociales. De esta manera se puede decir, que los estudiantes utilizan las tecnologías principalmente en el hogar (Vélez-Loor, Vallejo-Valdivieso \& Moya-Martínez, 2020).

La incorporación de TIC en la enseñanza, permite que el estudiante se transforme en participante activo y constructor de su propio aprendizaje y el profesor asume el rol de guía y facilitador, lo cual varía su forma de interactuar con sus educandos, la forma de planificar y de diseñar el ambiente de aprendizaje. Para (Pizarro-Chacón \& CorderoBadilla, 2013), estas herramientas tecnológicas permiten que el personal docente encuentre técnicas innovadoras para estimular la motivación del estudiantado y mejorar su aprendizaje con el uso de multimedia, hipertextos y otros sistemas interactivos, además de elegir aquellos recursos didácticos que mejor convengan a las distintas áreas del conocimiento de los estudiantes. (García-Cedeño, et al., 2020). Según (Calderón et al., 2015) la elección de herramientas tecnológicas no es un capricho o modismo sino una decisión pedagógica, que responde a nuestra intencionalidad educativa de enseñar y aprender destacando su carácter experimental, preparando al estudiante para que pueda resolver problemas de la vida diaria, pero realizando proyectos provocativos, motivantes y con desafíos para estudiantes de distintos niveles (Argandoña-Mendoza, et al., 2020).

De acuerdo con (García-Martín \& Cantón-Mayo, 2019), el uso que los estudiantes hacen de las tecnologías y el impacto de varias de herramientas tecnológicas en su rendimiento académico, permite que dichas herramientas facilitan la comunicación directa e individualizada, aumenta la confianza, su autoestima y la sensación de intimidad entre ellos y los demás. También hay que considerar la búsqueda de información en Internet esto implica que los estudiantes deben seleccionar fuentes adecuadas, y luego, extraer, organizar e integrar la información obtenida, de esta manera los educandos adquieren habilidades para la resolución de problemas. Al manejar redes sociales permite que el alumno mejore su participación en chats fortaleciendo las habilidades de comunicación y de interacción. 


\section{Referencial teórico}

Para (Riascos-Erazo, Quintero-Calvache \& Ávila-Fajardo, 2009), el acceso a las TIC debe ser de forma equitativa dentro de todos los niveles de educación, considerando que para alcanzar un nivel óptimo de inserción de las TIC en el aula es necesario contar con infraestructura tecnológica, capacitación docente, y apoyo en proyectos tecnológicos. Sin embargo, se debe tener en cuenta el nivel de conocimiento de los estudiantes sobre el manejo de las TIC dentro del proceso educativo, conscientes de que son nativos tecnológicos se asume que manejan cualquier herramienta tecnológica educativa, pero la realidad es otra debido a que los estudiantes utilizan y manejan únicamente programas de su preferencia como por ejemplo las redes sociales.

El aula tradicional de clase, se transforma en un aula innovadora mediante la implementación de las TIC, sin ser necesario estar en el clásico laboratorio de computación. De esta manera, se buscan formas efectivas de involucrar a docentes y estudiantes en la construcción de un aprendizaje significativo, propiciando interés y entusiasmo, con el fin resolver los complejos problemas de la educación actual.

Según un estudio de caso realizado por (Ortiz-Colón, Peñaherrera-León \& OrtegaTudela, 2012), presentan como resultados que dentro del aula parece que existen serios problemas para la dinámica de las clases cuando los equipos no se utilizan como un recurso integrado en el proceso de enseñanza y aprendizaje. Se llega a afirmar que los maestros no pueden ver lo que hacen porque las pantallas se lo impiden, otro de los inconvenientes que se presentan son los problemas técnicos de los equipos informáticos y la falta de un servicio técnico rápido y eficaz. A esto se suma la falta de conocimiento sobre el software disponible en los Pc de la institución, o incluso la ausencia de aplicaciones útiles para las distintas materias.

Otro problema a considerar es la deficiencia de formación de los docentes en cómo utilizar eficazmente (de forma integrada) las tecnologías en la enseñanza, por lo que afecta a la actitud de los profesores con relación a las TIC en la realidad del docente actual. Todo esto causa un efecto recursivo, el uso deficiente de las TIC en el aula. Como lado positivo de la investigación se establece, el cambio conceptual no fue una tarea sencilla en la historia de la humanidad y tampoco lo será para los estudiantes. 
Las investigaciones en didáctica de las ciencias muestran que, si los alumnos tienen oportunidad de construir hipótesis, diseñar experimentos, realizarlos y analizar cuidadosamente los resultados, será posible que superen la metodología del sentido común, al tiempo que se produzcan en ellos los cambios conceptuales que necesita el conocimiento científico.

Por otro lado, (Martínez-Figueroa \& Raposo-Rivas, 2006), en su estudio de caso en la facultad de Psicopedagogía habla de la importancia que hoy en día tienen las TIC en prácticamente todos los ámbitos profesionales, constatado el vacío formativo existente sobre esta temática en el plan de estudios de la titulación de Psicopedagogía, hacemos un estudio longitudinal con la finalidad de evaluar las competencias transversales y específicamente tecnológicas que poseen los estudiantes de titulación. Así, con tales precedentes y siguiendo una metodología cuantitativa por medio de la técnica de la encuesta, se elaboró un cuestionario y se obtuvo un resultado en donde el $88 \%$ de los estudiantes utiliza casi en nada las TIC para hacer tutorías.

De este modo, los nuevos profesionales se titulan sin dar el uso adecuado y correcto de las TIC, es por esto que se debe interactuar en clase docente y estudiantes con el manejo de las TIC, si bien es cierto se da ciertos problemas ya que los educandos no saben manejar herramientas informáticas, se puede decir que parte de este problema radica en el currículo nacional ecuatoriano, que rige en el artículo 4 del acuerdo ministerial Nro. MINEDUC-ME-2016-00020-A, emitido por el (Ministerio de Educación, 2020) debido que no existe la asignatura de Informática o TIC, en ningún nivel de educación, resultando así un limitante en la calidad de la educación, es decir los alumnos no desarrollan las habilidades para manejar dichos aplicativos.

Mientras se incluyen a cabalidad el uso de las TIC en el proceso enseñanza aprendizaje, tanto docentes como estudiantes se acoplan al uso de las TIC en el aula, y se adaptan a los nuevos cambios de educación, surge una pandemia mundial denominada Coronavirus o COVID-19. Los coronavirus (COVID-19) son una amplia familia de virus, algunos tienen la capacidad de transmitirse de los animales a las personas. Según el (Ministerio de Salud Pública, 2020), producen cuadros clínicos que van desde el resfriado común hasta enfermedades más graves, como ocurre con 
el coronavirus que causó el síndrome respiratorio agudo grave (SARS-CoV) y el coronavirus causante del síndrome respiratorio de Oriente Medio (MERS-CoV). A partir de esta situación mundial, La (Organización Mundial de la Salud OMS, 2020) está colaborando estrechamente con expertos mundiales, gobiernos y asociados para ampliar rápidamente los conocimientos científicos sobre este nuevo virus, rastrear su propagación y virulencia y asesorar a los países y las personas sobre la medida para proteger la salud y prevenir la propagación del brote.

En Ecuador, el Gobierno Nacional mediante Decreto Ejecutivo № 1017 emitido por el (Ministerio de Defensa Nacional, 2020), la Declaratoria de Estado de Excepción por calamidad pública en todo el territorio nacional, una vez que la Organización Panamericana de la Salud declarara la pandemia del COVID-19, disponiendo medidas relacionadas a la movilización, libre tránsito, cuarentena obligatoria de los ciudadanos, toque de queda, suspensión de la jornada laboral presencial de todos los trabajadores del sector público como del sector privado, con las excepciones establecidas en el citado Decreto.

De este modo, toda la sociedad pasa a estar aislada en su domicilio, fomentándose así el teletrabajo y la teleeducación. De la misma manera, en cadena nacional de radio y televisión trasmitida el día viernes 10 de abril de 2020, el Señor presidente de la República - Lcdo. Lenin Moreno, exhortó a que se buscarán soluciones para los estudiantes, tanto del sistema fiscal como del particular, con el objetivo de que no abandonen la escuela, en el marco de la emergencia sanitaria por el COVID-19.

EI (Ministerio de Educación, 2020), emite un acuerdo ministerial ACUERDO Nro. MINEDUC-MINEDUC-2020-00020-A, en el cual manifiesta, "Disponer la suspensión de clases en todo el territorio nacional para todas las instituciones educativas públicas, fiscomisionales y particulares del régimen Sierra - Amazonía 2019-2020, en todas sus jornadas y modalidades, hasta el 30 de abril de 2020, " y "Disponer al personal administrativo y docente del Sistema Nacional de Educación, continuar ejecutando sus labores mediante la modalidad de teletrabajo."

Ante la situación que atraviesa el país, con el alto índice de contagios de COVID-19, en todas sus provincias la Ministra de Educación Monserrat Cremer mediante entrevista televisiva manifestó "Contemplamos que este escenario educativo será 
fácilmente hasta finales de junio y que por lo tanto estamos preparados para terminar así el año escolar, hasta el 30 de junio", de modo virtual.

De esta manera docentes y estudiantes se ven obligados a utilizar las TIC para el proceso enseñanza - aprendizaje, buscando medios y estrategias para obtener una óptima comunicación entre profesores y educandos, propiciando la investigación, uso y manejo de las diferentes herramientas tecnológicas tales como: aulas virtuales, plataformas educativas, Blogs, Redes Sociales, Webinar entre otros.

Para (Almansa, Fonseca \& Castillo, 2013), los adolescentes tener un perfil en Facebook representa administrar su personalidad. Crear un perfil en Facebook y asignar contenido e historias en la interfaz de esta red social es un acto de creación de un ser en un entorno digital, generado así una serie de códigos nuevos de escritura, que evitan la gramática y las reglas ortográficas, es decir que obedecen a otras condiciones como por ejemplo la velocidad de escritura y especialmente las estéticas digitales.

Por consiguiente, la presente investigación tiene como objetivo identificar el uso y manejo de herramientas tecnológicas en el proceso enseñanza - aprendizaje durante la pandemia de Coronavirus COVID-19 en Ecuador mediante el análisis de la utilización de recursos TIC por docentes y estudiantes para alcanzar una educación significativa y proponer el uso de la Red Social más utilizada como lo es Facebook con fines pedagógicos mediante el uso correcto de la misma para emplearla como herramienta educativa durante la pandemia de Coronavirus COVID - 19.

\section{MÉTODO}

De esta manera haciendo frente a la problemática mundial que se está viendo, se planteó la investigación de un estudio de caso con 58 docentes de diferentes establecimientos educativos del cantón Azogues, y con 200 estudiantes de primero y segundo de bachillerato de la Unidad Educativa Particular "La Providencia" de Azogues, focalizándose desde la vertiente cuantitativa de investigación (AldanaZavala, 2019).

Por tanto, según (Argudo-Tello, Erazo-Álvarez, \& Narváez-Zurita, 2019), esta investigación es descriptiva no experimental, es de carácter transversal en la cual se 
tomó en un solo cohorte la muestra de datos, realizando encuestas a docentes y estudiantes mediante Google Drive, la encuesta a docentes consta de 8 preguntas y para los estudiantes una encuesta diferente que consta de 5 preguntas, con el fin de conocer y analizar sobre el uso y manejo de las TIC en el proceso enseñanza aprendizaje desde los dos puntos de vista (Peñafiel-Sacoto, Erazo-Álvarez, PozoCabrera, \& Narváez-Zurita, 2020).

En dichas encuestas se obtuvo datos cualitativos para examinarlos mediante un cuadro comparativo sobre el uso de las herramientas tecnológicas y cuantitativos para obtener el porcentajes de sus respuestas frente al uso de las TIC de docentes y estudiantes durante la pandemia de COVID-19, luego los datos de estas encuestas fueron validadas en el Software SPSS, el cálculo de la fiabilidad de las dos encuestas se realizó a través del coeficiente de correlación alfa de Cronbach en la cual de la encuesta a estudiantes se obtuvo el $0.703 \%$ y en la encuesta a docentes se obtuvo el 0.895\%, y el análisis estadístico descriptivo mediante Chi - cuadrado.

\section{RESULTADOS}

En medio de esta pandemia de COVID-19, la teleeducación se está llevando a cabo mediante múltiples opciones, pero he aquí un comparativo de los resultados obtenidos en las encuestas realizada a los docentes y estudiantes sobre la realidad que está viendo en estos momentos. Se analiza el uso de las TIC para el proceso enseñanza - aprendizaje, en el cual se puede observar que tanto docentes y estudiantes manifiestan que el uso de las TIC en el aula fue de nivel medio.
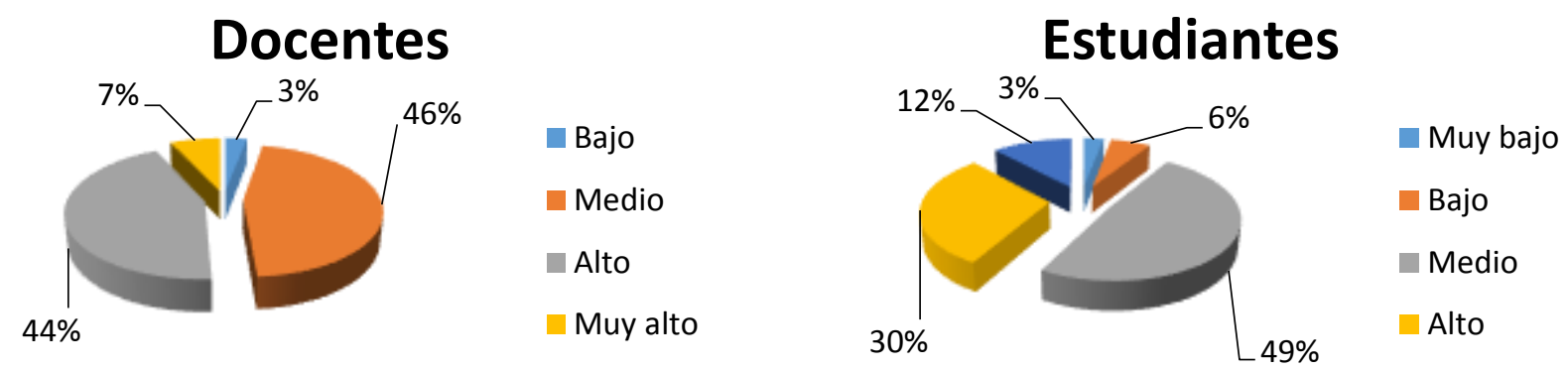

Figura 1 Comparación nivel de empleo de las TIC en las aulas docente y estudiantes. Fuente: Elaboración Propia 
Mediante la siguiente tabla podemos observar que en clases presenciales los estudiantes usaban medianamente las TIC, mientras que con este cambio a las clases virtuales se han visto obligados a usarlas.

\section{Tabla 1}

Relación entre estudiantes y el uso de las TIC durante la pandemia del COVID-19

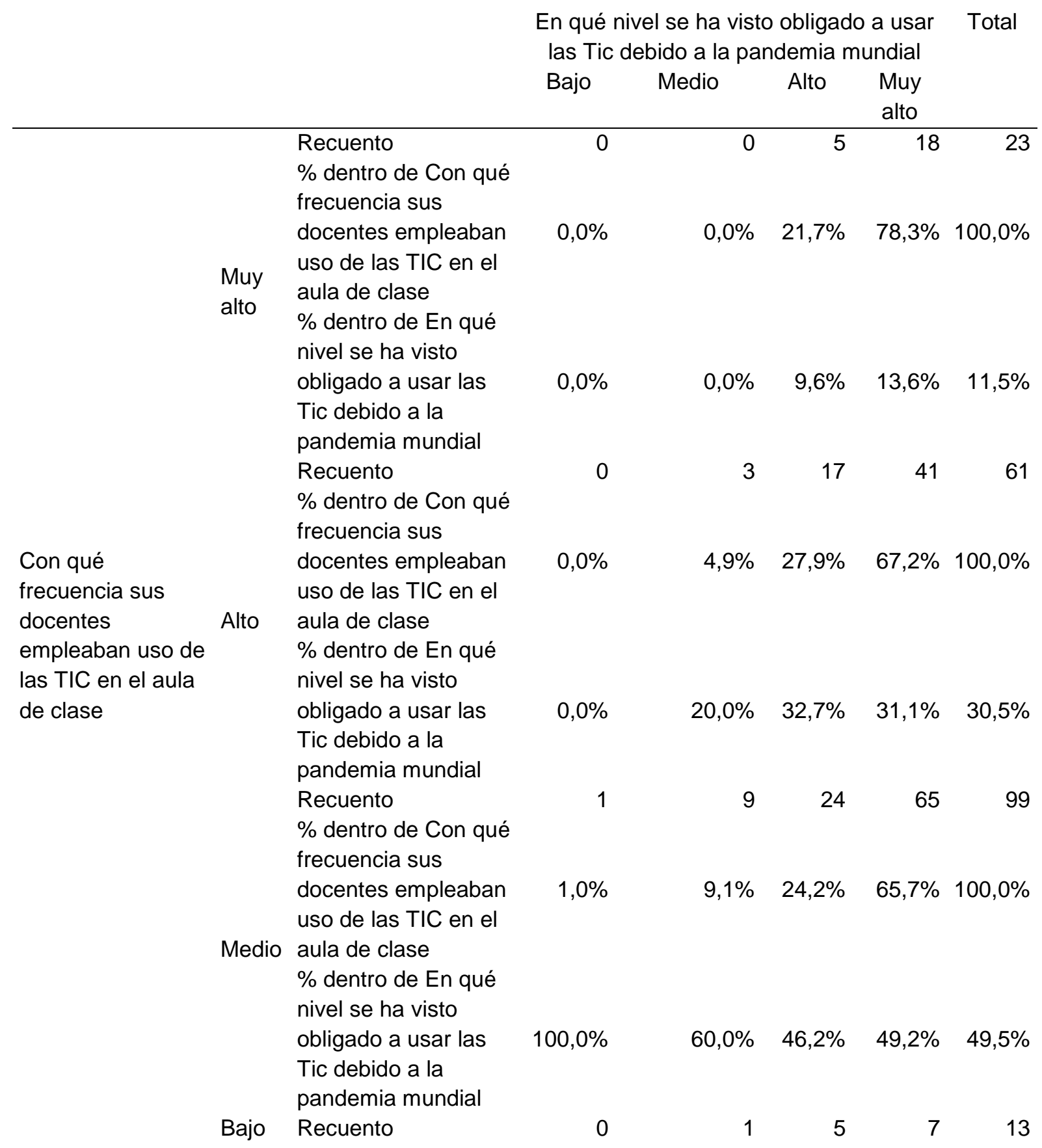




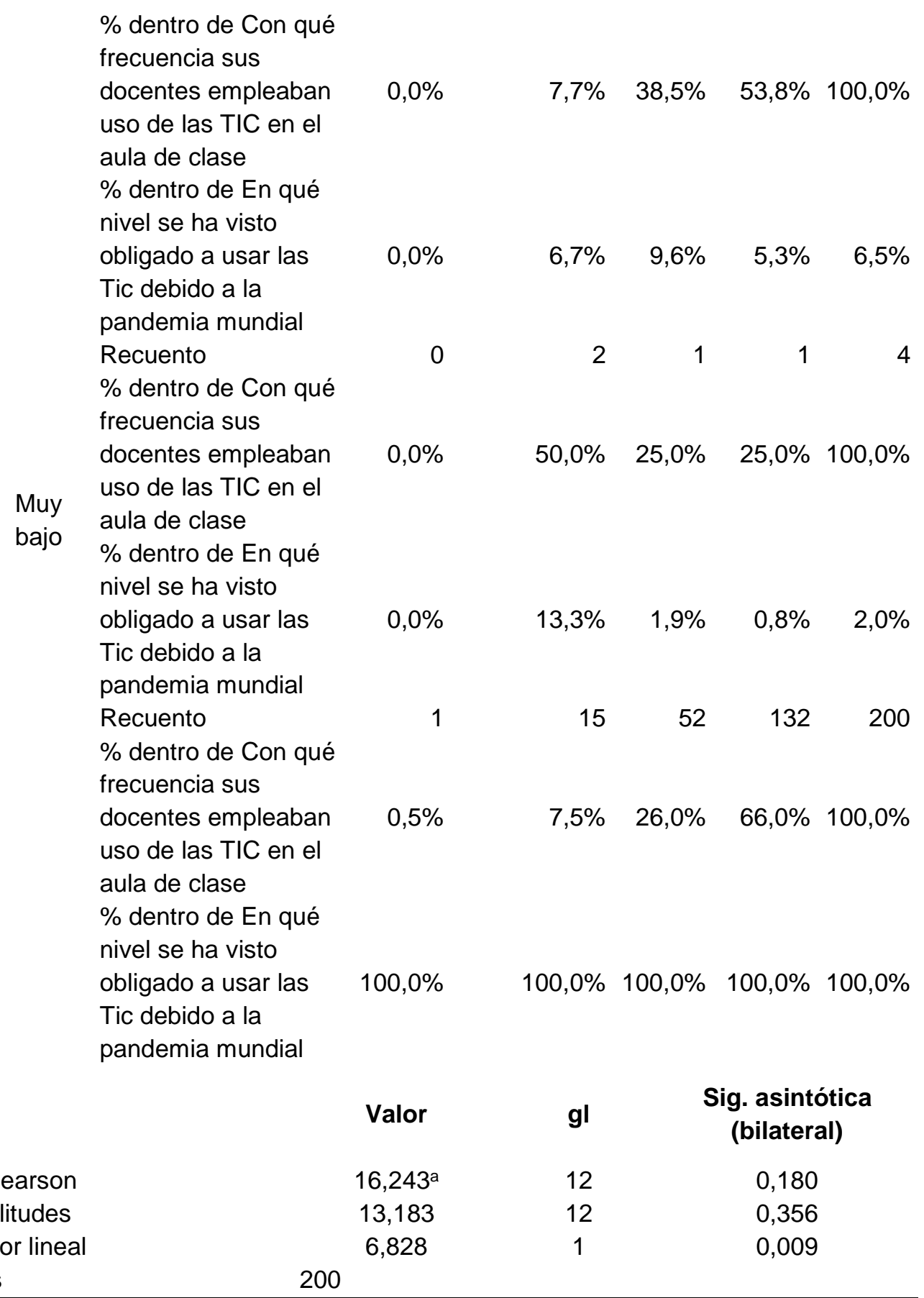

Chi-cuadrado de Pearson Razón de verosimilitudes Asociación lineal por lineal $\mathrm{N}$ de casos validos $\%$ dentro de Con qué frecuencia sus docentes empleaban uso de las TIC en el $\%$ dentro de En qué nivel se ha visto obligado a usar las pandemia mundia Recuento frecuencia sus (a) ula de clase nivel se ha visto pandemia mundia

Fuente: Elaboración Propia

Haciendo un análisis estadístico entre docentes y estudiantes ante esta nueva forma de enseñar online, se puede observar que los docentes y estudiantes manifiestan que la enseñanza impartida por estas herramientas tecnológicas tiene un nivel alto debido que las clases son claras y precisas. 

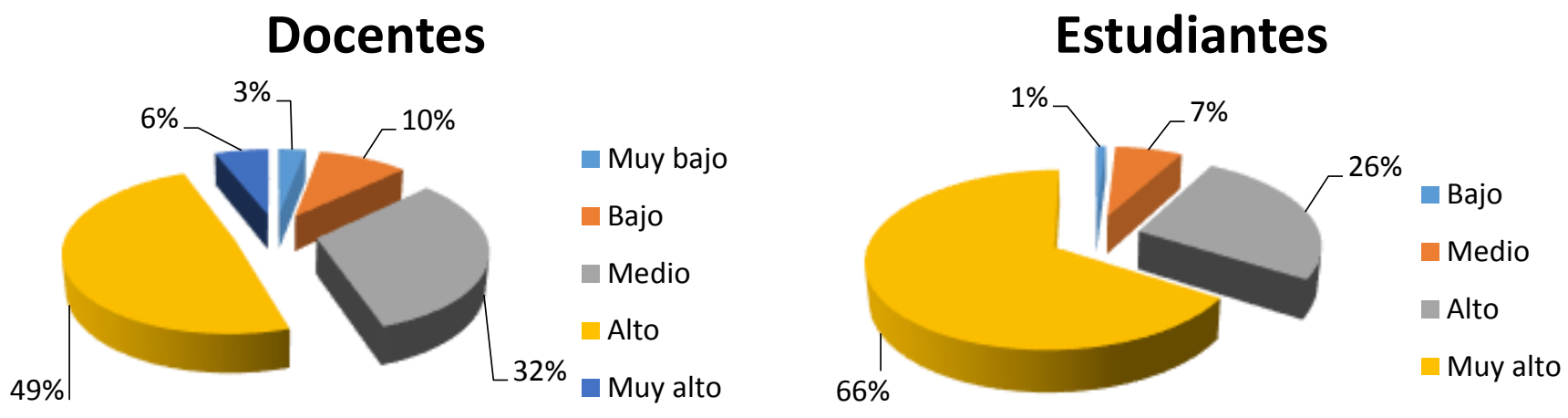

Figura 2. Comparación del uso de las TIC en la enseñanza durante la pandemia del COVID-19. Fuente: Elaboración Propia

Analizando la hipótesis de la teleeducación permite un aprendizaje significativo para docente y estudiantes.

Docentes

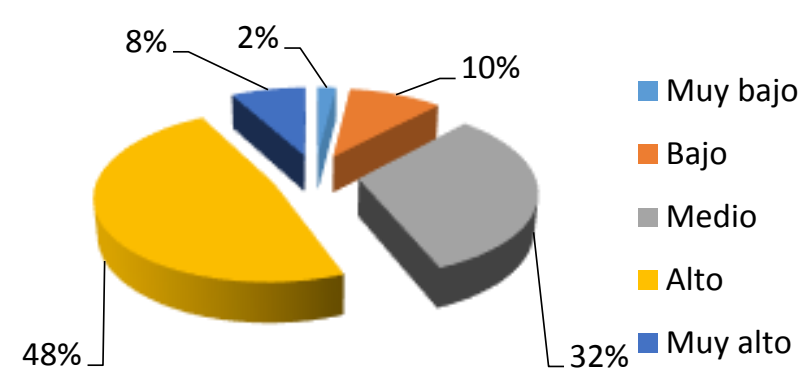

Estudiantes

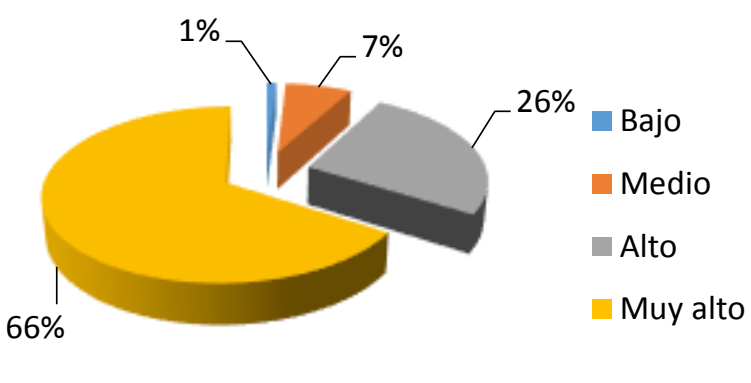

Figura 3. Comparación del aporte de la teleeducación para el aprendizaje significativo. Fuente: Elaboración Propia. 
Planteando la siguiente hipótesis los docentes no están completamente preparados tecnológicamente para dar clases online, sin embargo, está nueva enseñanza permite a los estudiantes tener una formación estudiantil durante la pandemia.

\section{Docentes}

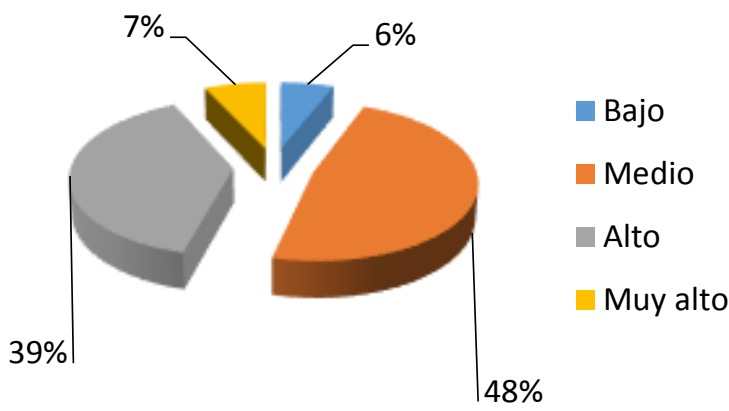

\section{Estudiantes}

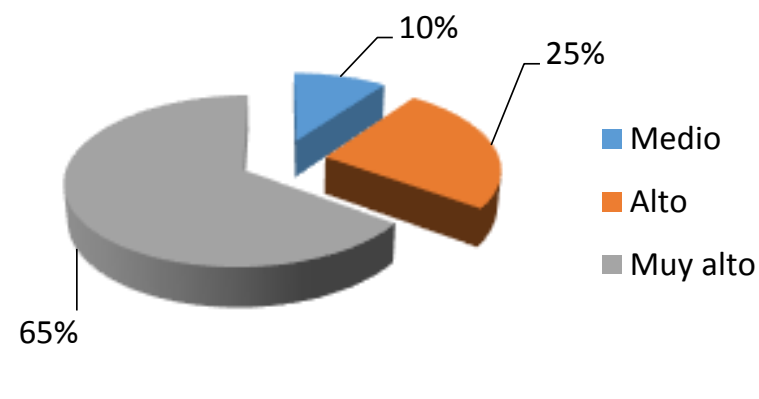

Figura 4. Comparación de la importancia de impartir clases online para continuar con la formación estudiantil durante la pandemia del COVID-19. Fuente: Elaboración propia

Según la hipótesis que los docentes implementan las TIC en el proceso de enseñanza medianamente, en la cuarentena los docentes se han visto obligados a manejar las TIC como instrumento para la enseñanza.

Tabla 2. Relación docente y uso de las TIC durante la pandemia de COVID-19

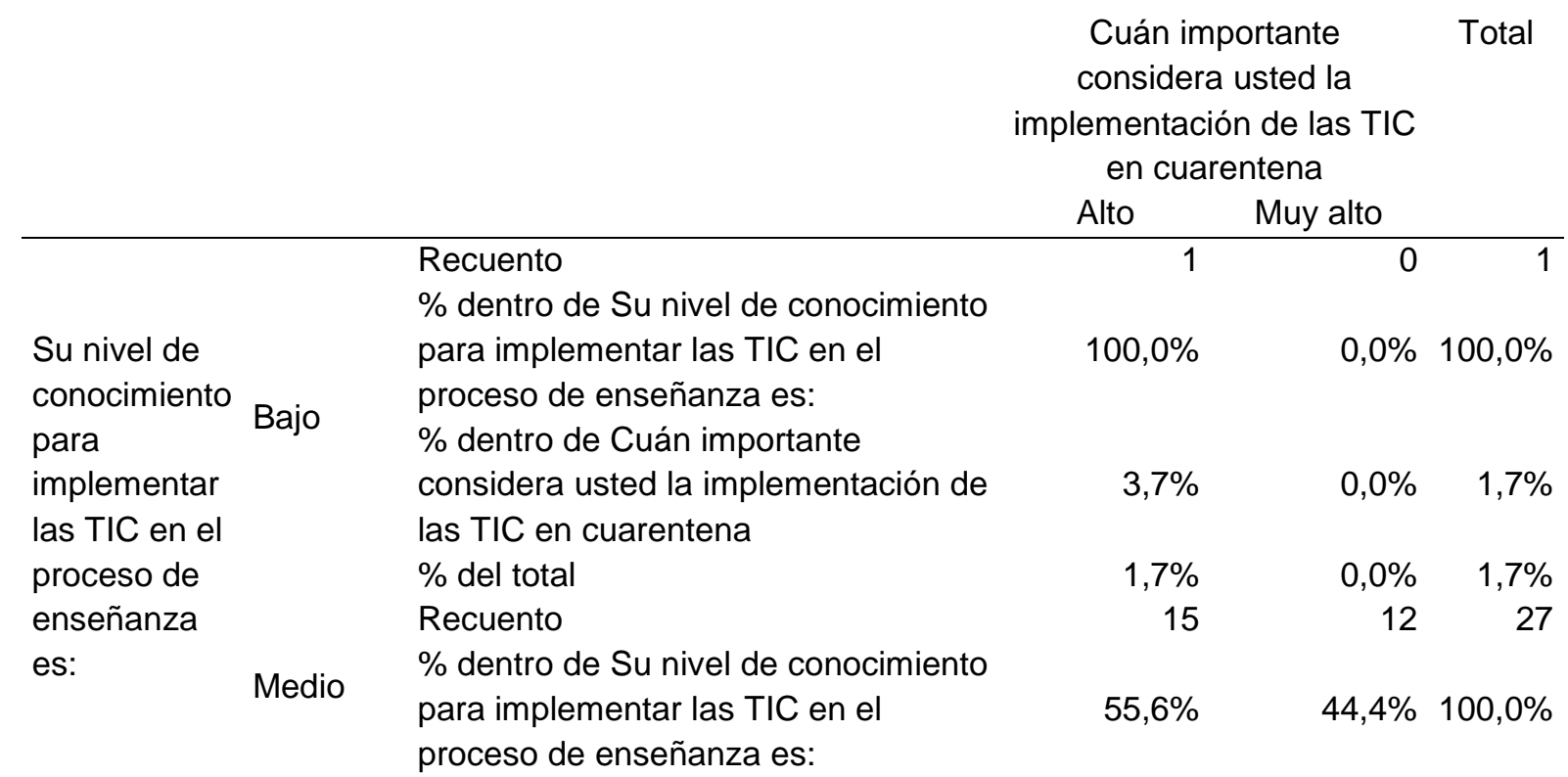


Paola Cecilia Pinos-Coronel; Darwin Gabriel García-Herrera; Juan Carlos Erazo-Álvarez; Cecilia Ivonne Narváez-Zurita

$\%$ dentro de Cuán importante considera usted la implementación de las TIC en cuarentena

$\%$ del total

Recuento

$\%$ dentro de Su nivel de conocimiento para implementar las TIC en el

Alto proceso de enseñanza es:

$\%$ dentro de Cuán importante considera usted la implementación de las TIC en cuarentena

$\%$ del total

Recuento

$\%$ dentro de Su nivel de conocimiento para implementar las TIC en el

Muy alto proceso de enseñanza es:

$\%$ dentro de Cuán importante considera usted la implementación de las TIC en cuarentena

$\%$ del total

Recuento

$\%$ dentro de Su nivel de conocimiento

para implementar las TIC en el

Total

\begin{tabular}{|c|c|c|}
\hline $55,6 \%$ & $38,7 \%$ & $46,6 \%$ \\
\hline $25,9 \%$ & $20,7 \%$ & $46,6 \%$ \\
\hline 11 & 15 & 26 \\
\hline $42,3 \%$ & $57,7 \%$ & $100,0 \%$ \\
\hline $40,7 \%$ & $48,4 \%$ & $44,8 \%$ \\
\hline $19,0 \%$ & $25,9 \%$ & $44,8 \%$ \\
\hline 0 & 4 & 4 \\
\hline $0,0 \%$ & $100,0 \%$ & $100,0 \%$ \\
\hline $0,0 \%$ & $12,9 \%$ & $6,9 \%$ \\
\hline $0,0 \%$ & $6,9 \%$ & $6,9 \%$ \\
\hline 27 & 31 & 58 \\
\hline $46,6 \%$ & $53,4 \%$ & $100,0 \%$ \\
\hline $100,0 \%$ & $100,0 \%$ & $100,0 \%$ \\
\hline & $53,4 \%$ & $100,0 \%$ \\
\hline
\end{tabular}

\begin{tabular}{cccc}
\hline & Valor & gl & $\begin{array}{c}\text { Sig. asintótica } \\
\text { (bilateral) }\end{array}$ \\
\hline Chi-cuadrado de Pearson & $5,700^{\mathrm{a}}$ & 3 & 0,127 \\
Razón de verosimilitudes & 7,607 & 3 & 0,055 \\
Asociación lineal por lineal & 4,688 & 1 & 0,030 \\
N de casos validos & 58 & & \\
\hline
\end{tabular}

Fuente: Elaboración Propia

En lo que respecta a las herramientas tecnológicas utilizadas para la teleeducación durante la pandemia COVID-19 se presenta que las más utilizadas son: WhatsApp, Zoom, Utilitarios Office, plataforma institucional y videos tutoriales en YouTube. 


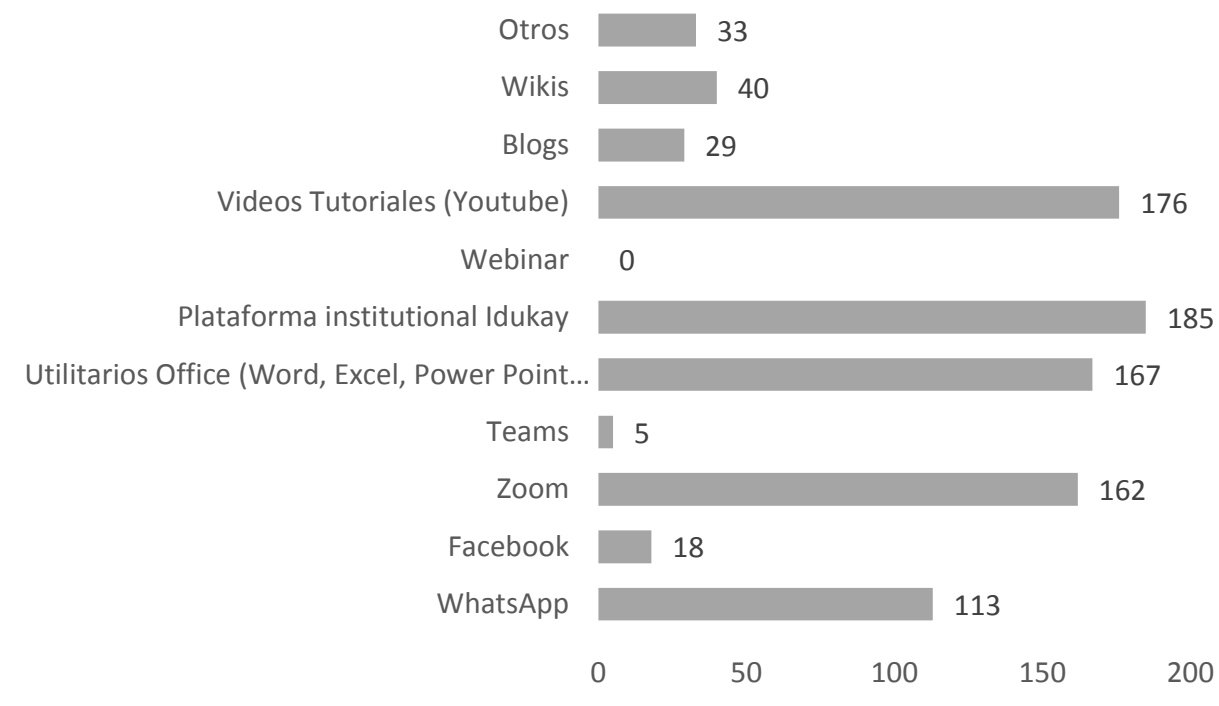

Figura 5. Herramientas tecnológicas utilizadas para el proceso enseñanza aprendizaje durante la pandemia del COVID-19. Fuente: Elaboración propia

Como se puede observar los resultados demuestran que los docentes en las clases presenciales usaban las TIC de forma aleatoria y no estaban completamente preparados tecnológicamente para tener un desempeño al 100\% impartiendo sus clases por medio de las TIC, pero al presentarse la suspensión de clases presenciales y tomar la medida de clases online los maestros se han visto obligados a conocer, investigar y prepararse para usar y manejar las TIC como herramienta clave para el proceso enseñanza - aprendizaje.

Si bien los estudiantes manejan tecnología, pero no siempre con fines educativos, al tener clases virtuales se han visto obligados a conocer y usar nuevas herramientas tecnológicas que les permita adaptarse a esta nueva modalidad para alcanzar un aprendizaje significativo. Debido a la presión de docentes y estudiantes por manejar las TIC para el proceso educativo con el fin de alcanzar una teleeducación con éxito, no se ha considerado que las redes sociales, en este caso Facebook se la puede mirar desde un punto de vista educativo que permita interactuar profesor - alumno para lograr una mejor interacción entre los mismos. 


\section{PROPUESTA}

Lo primero que se debe plantear es una capacitación a docentes, estudiantes y padres de familia sobre el uso correcto de las herramientas tecnológicas por cuanto como se presenta en la figura 5 estas aplicaciones facilitan las clases virtuales, los docentes hacen lo posible por cumplir con esta actividad, pero por parte de estudiantes existe mal uso de la tecnología y pérdida de valores convirtiéndose en un problema al momento que los maestros llevan a cabo su clase, de tal motivo no se puede alcanzar una enseñanza de forma eficiente, eficaz, al impartir clases virtuales.

Por otra parte, se plantea el uso pedagógico de Facebook, que es una red social que se lanzó en 2004. Es una aplicación que permite acceder desde cualquier dispositivo móvil, tiene millones de usuarios a nivel mundial y les ofrece a sus usuarios una variedad de formas beneficiosas para interactuar con amigos y con el mismo sitio. Esta red social brinda la posibilidad de que los usuarios estén contactos con aquellos que los rodean y con lo que pasa en el mundo en cualquier momento, también es una forma de invitar a otros a eventos.

La función principal de Facebook es conectar a amigos y familiares. Utilizando las herramientas de búsqueda y sugerencias, es fácil encontrar amigos actuales y de hace mucho tiempo. Esto te habilita a compartir contenido con amigos y ver el contenido que ellos publican también. Este puede ser una publicación en tu página (conocido como Muro) o fotos, videos e información personal. Tienes la opción de compartir mucho o poco con tus amigos. Estas interacciones te permiten estar en contacto con las personas, a veces con alguien con quien no estarías conectado de otra manera regularmente.

Entre las diversas funcionalidades que nos permite Facebook está: Facebook Live, Facebook Watch, Messenger Facebook, para obtener una interacción dinámica entre sus miembros. Facebook presenta ventajas por ejemplo cuando descargamos la aplicación se crea un acceso directamente en la pantalla principal del dispositivo, donde solo veras los mensajes que te lleguen de tus contactos de Facebook, ahorrando espacio de almacenamiento. En cuanto se refiere a mensajería instantánea permite enviar fotos, audios, realizar videos llamadas, archivos, enviar videos, tomar fotos en el momento. Se presenta menú de stickers y emoji, permite cambiar los 
colores del chat, Facebook brinda servicio de soporte técnico y reportes de problemas, además permite elegir estar en línea o no.

Por todo lo antes mencionado, y muchas actualizaciones que presenta hoy en día Facebook durante la pandemia de Covid-19, se realiza una propuesta educativa con el fin de usar esta herramienta tecnológica muy conocida para fines educativos y lograr una buena interacción entre docente - estudiante para alcanzar un aprendizaje significativo, una enseñanza de calidad dentro de la denominada teleeducación. En la siguiente figura se presenta un esquema del uso educativo de Facebook.
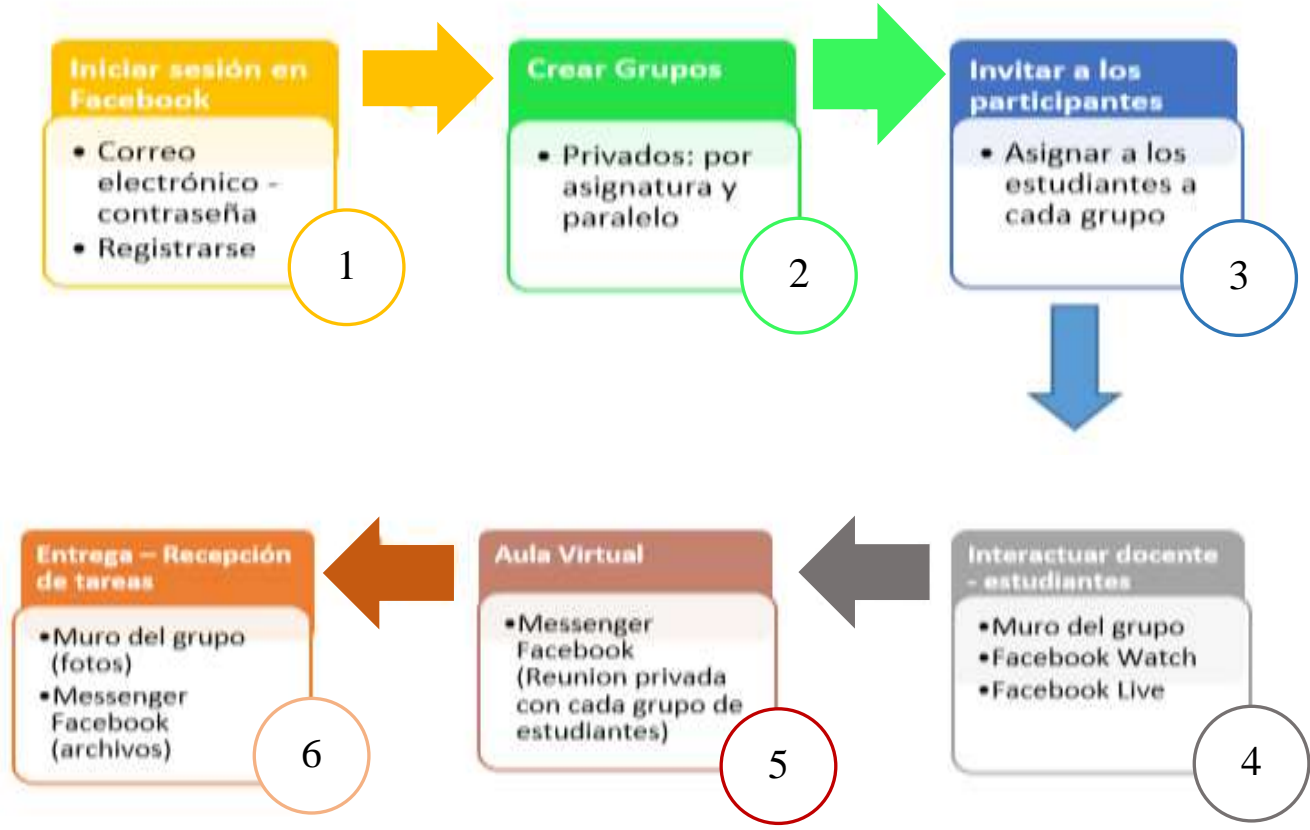

Figura 6. Facebook como herramienta tecnológica educativa. Fuente: Elaboración propia

1. Iniciar sesión en Facebook: tener una cuenta - perfil activo en Facebook, en caso de ya tenerlo ingresar correo electrónico y contraseña, en el caso de no tener un perfil, registrarse para poder acceder.

2. Crear Grupos: para ello damos clic en Grupos y clic en crear podemos hacerlo por asignatura y por paralelos, dependiendo de la necesidad del docente, configurando las características que se consideren necesarios tales como: nombre, foto de portada, privacidad (público o privado), entre otros. 
3. Invitar a los participantes: Se lo puede hacer mediante invitación directa buscando a la persona, enviando el enlace del grupo, compartiendo por Messenger, enviar por correo.

4. Interactuar docente - estudiantes: Esta red social nos permite realizar comentarios, consultas y opiniones, cargar videos mediante la opción de Facebook Watch en ella se puede enlazar un video tutorial de YouTube o un video grabado por el docente exponerlo a los estudiantes e interactuar con ellos, también lo podemos hacer una conexión más real mediante Facebook Live la misma que permite dar la clase en vivo e interactuar con los participantes mediante comentarios o reacciones.

5. Aula Virtual: Esta actividad lo podemos hacer mediante Facebook Messenger el cual nos brinda la opción de video llamada que permite realizar las denominadas aulas virtuales, creando salas desde cualquier dispositivo, lo podemos realizar enviando el enlace de la reunión o para las personas que poseen cuenta de Facebook es decir configurar la privacidad de la reunión, esta función permite hasta 50 participantes. Una buena opción también para lecciones orales, conversatorios y debates.

6. Entrega - Recepción de tareas: Para poder receptar las tareas por los estudiantes lo podemos hacer mediante la publicación de fotos de la tarea en el muro del grupo, o si es en archivo lo podemos receptar por Messenger de Facebook.

\section{CONCLUSIONES}

Mediante los resultados obtenidos podemos decir que los docentes no estaban preparados tecnológicamente, por cuanto usaban las TIC esporádicamente para impartir sus clases, sin embargo, debido a la pandemia que se está viviendo a nivel mundial los docentes se han visto obligados a prepararse más en el uso y manejo herramientas tecnológicas, con el fin de buscar brindar una enseñanza de calidad.

De la misma manera los estudiantes, pese que son nativos tecnológicos no usaban aplicaciones tecnológicas educativas sino solo las de su agrado, de esta manera los 
educandos también han tenido que aprender, usar y manejar herramientas tecnológicas, para continuar con su formación estudiantil.

Sin embargo, en esta emergencia sanitaria que se está atravesando se puede decir que tanto docentes y estudiantes han hecho su mejor esfuerzo para estar tecnológicamente activos para interactuar en el proceso enseñanza - aprendizaje y obtener un aprendizaje significativo, y poder culminar el año de la mejor manera.

Tomando en cuenta que docentes y estudiantes no estuvieron preparados para abandonar las aulas de clase de forma presencial y pasar a modo virtual, sumando esfuerzos de parte a parte para alcanzar una buena educación y comprensión de la misma.

Para ello, entre las herramientas tecnológicas más utilizadas durante esta pandemia están: WhatsApp, Zoom, Utilitarios Office, plataforma institucional Idukay y videos tutoriales en YouTube, las cuales son un soporte primordial en la teleeducación para que tanto docentes y estudiantes tengan una comunicación directa en el proceso enseñanza - aprendizaje.

Por tanto, se concluye que las TIC juegan un papel importante en el proceso enseñanza - aprendizaje ya que permite pasar del modelo pedagógico del constructivismo al Conectivismo, siendo las TIC, la pieza fundamental en la enseñanza durante la cuarentena en la pandemia COVID - 19, ya que permite la conexión e interacción maestro - estudiante.

De este modo, se puede demostrar que el uso correcto y adecuado de una red social, permite y facilita el proceso de enseñanza - aprendizaje, convirtiendo en un aprendizaje para la vida, siendo este llamativo y de interés para los estudiantes ya que pueden hacerlo desde cualquier dispositivo móvil.

\section{FINANCIAMIENTO}

No monetario.

\section{AGRADECIMIENTO}

Al personal docente y a los estudiantes de la Unidad Educativa Particular "La Providencia" de Azogues, por el apoyo en la realización de la investigación. 


\section{REFERENCIAS}

Argandoña-Mendoza, M., García-Mejía, R., Ayón-Parrales, E., \& ZambranoZambrano, Y. (2020). Investigación e innovación educativa: Reto escolar por COVID-19 en el Ecuador. [Educational research and innovation: School challenge for COVID-19 in Ecuador]. EPISTEME KOINONIA, 3(5), 162-182. http://dx.doi.org/10.35381/e.k.v3i5.726

Aldana-Zavala, J. J. (2019). La competencia epistemológica en el investigador social universitario venezolano. [Epistemological competence in the Venezuelan university social researcher]. Praxis, 15(1), 103-115. https://doi.org/10.21676/23897856.3091

Almansa-Martínez, A., Fonseca, O., \& Castillo-Esparcia, A. (2013). Social networks and young people. Comparative study of Facebook between Colombia and Spain. [Redes sociales y jóvenes. Uso de Facebook en la juventud colombiana y española]. Comunicar, 40, 127-135. https://doi.org/10.3916/C40-2013-03-03

Argudo-Tello, K., Erazo-Álvarez, J., \& Narváez-Zurita, C. (2019). Evaluación de Control Interno en Riesgos Estratégicos para la Dirección de Planificación de la Universidad de Cuenca. [Evaluation of Internal Control in Strategic Risks for the Planning Direction of the University of Cuenca]. Revista Arbitrada Interdisciplinaria Koinonía, 4(1), 67-96. http://dx.doi.org/10.35381/r.k.v4i1.372

Calderón, S., Núñez, P., Di Laccio, J., lanelli, L., \& Gil, S. (2015). Aulas-laboratorios de bajo costo, usando TIC. [Low-cost classrooms-laboratories, using ICT]. Revista Eureka sobre Enseñanza y Divulgación de las Ciencias, 12(1), 212226. Recuperado de https://n9.cl/fOgt

Creamer, M. (2020). Plan Educativo Covid-19 se presentó el 16 de marzo. [Covid-19 Educational Plan was presented on March 16]. Recuperado de https://url2.cl/fu54M

García-Cedeño, G., Vélez-Loor, M., Franco-Zambrano, C., \& Ormaza-Bermello, M. (2020). Educación por competencias: Un reordenamiento curricular durante emergencia escolar por COVID-19. [Competency Education: A Curricular Rearrangement During School Emergency by COVID-19]. EPISTEME KOINONIA, 3(5), 221-235. http://dx.doi.org/10.35381/e.k.v3i5.770

García-Martín, S., \& Cantón-Mayo, I. (2019). Use of technologies and academic performance in adolescent students. [Uso de tecnologías y rendimiento académico en estudiantes adolescentes]. Comunicar, 59, 73-81. https://doi.org/10.3916/C59-2019-07 
Martínez-Figueroa, M., \& Raposo-Rivas, M. (2006). Las TIC en manos de estudiantes universitarios. [ICT in the hands of university students]. Revista Latinoamerica de Tecnología Educativa, 5(2), 165-176. Recuperado de https://n9.cl/f0gt

Ministerio de Defensa Nacional. (2020). Decreto Presidencial № 1017. [Presidential Decree No. 1017]. Recuperado de https://n9.cl/xx68

Ministerio de Educacion (2020). Acuerdo Nro. MINEDUC-MINEDUC-2020-00020-A. [agreement No. MINEDUC-MINEDUC-2020-00020-A.]. Recuperado de https://n9.cl/9pfz

Ministerio de Salud Pública (2020). Coronavirus COVID 19. [Coronavirus COVID 19]. Recuperado de https://n9.cl/p67ee

Morffe, A. (2010). Las TIC como herramientas mediadoras del aprendizaje significativo en el pregrado: una experiencia con aplicaciones telemáticas gratuitas. [ICT as mediating tools for meaningful learning in undergraduate: an experience with free telematic applications]. Revista de Artes y Humanidades UNICA, 11 (1), 200-219. Recuperado de https://n9.cl/siqsh

OMS (2020). Brote de enfermedad por Coronavirus (COVID-19). [Coronavirus disease outbreak (COVID-19)]. Recuperado de https://n9.cl/psbv

Orduz, R. (2012). Sociedad del conocimiento y tecnologías de la información. [Knowledge society and information technologies]. En Orduz, R. (Ed). Aprender y Educar con las tecnologias del Siglo XXI. [Learn and Educate with the technologies of the XXI Century]. (pp. 9-12). Bogotá: Corporación Colombia Digital.

Ortiz-Colón, A. M., Peñaherrera-León, M., \& Ortega-Tudela, J. M. (2012). Percepciones de profesores y estudiantes sobre las TIC. Un estudio de caso. [Teachers 'and students' perceptions of ICT. A case study]. Edutec. Revista Electrónica de Tecnología Educativa, (41), a210. https://doi.org/10.21556/edutec.2012.41.352

Organización de las Naciones Unidas para la Educación, la Ciencia y la Cultura (2013). Enfoques estratégicos sobre las TICS en educación en América Latina y el Caribe. [Strategic approaches to ICTs in education in Latin America and the Caribbean]. Recuperado de https://n9.cl/0gi1

Peñafiel-Sacoto, J., Erazo-Álvarez, J., Pozo-Cabrera, E., \& Narváez-Zurita, C. (2020). La fundamentación y la motivación como habilitantes de la prisión preventiva. [The rationale and motivation as enablers of preventive detention]. IUSTITIA SOCIALIS, 5(8), 465-492. http://dx.doi.org/10.35381/racji.v5i8.586 
Pizarro-Chacón, G, \& Cordero-Badilla, D. (2013). Las TIC: Una herramienta tecnológica para el desarrollo de las competencias lingüísticas en estudiantes universitarios de una segunda lengua. [ICT: A technological tool for the development of language skills in university students of a second language]. Revista Electrónica Educare, 17(3), 277-292. Recuperado de https://n9.cl/uv9o

Quevedo-Álava, R., Corrales-Moreno, L., Palma-Delgado, G., \& Mendoza-Suárez, G. (2020). Psicopedagogía y TIC en período de COVID-19. Una reflexión para el aprendizaje significativo. [Psychopedagogy and ICT in the COVID-19 period. A Reflection for Meaningful Learning]. EPISTEME KOINONIA, 3(5), 202-220. http://dx.doi.org/10.35381/e.k.v3i5.769

Riascos-Erazo, Sandra Cristina, Ávila-Fajardo, Gloria Patricia, \& Quintero-Calvache, Diana María. (2009). Las TIC en el aula: percepciones de los profesores universitarios. [ICT in the classroom: perceptions of university professors]. Educación y Educadores, 12(3), 133-157. Recuperado de https://n9.cl/uukf

Saltos-Cedeño, A., Vallejo-Valdivieso, P., \& Moya-Martínez, M. (2020). Innovación en educación matemática de básica superior durante el confinamiento por COVID19. [Innovation in higher basic mathematics education during confinement by COVID-19]. EPISTEME KOINONIA, 3(5), 142-161. http://dx.doi.org/10.35381/e.k.v3i5.723

Vélez-Loor, M., Vallejo-Valdivieso, P., \& Moya-Martínez, M. (2020). Recursos didácticos virtuales en proyectos de ciencias naturales en período de confinamiento por COVID-19. [Virtual teaching resources in natural science projects in confinement period by COVID-19]. EPISTEME KOINONIA, 3(5), 183-201. http://dx.doi.org/10.35381/e.k.v3i5.760 